\title{
ÍNDICE INTRAURBANO DE BEM-ESTAR EM SÃO LUÍS-MA, BRASIL
}

\author{
Paulo Roberto Mendes Pereira \\ Universidade Estadual Paulista, Faculdade de Ciência e Tecnologia \\ Programa de Pós-Graduação em Geografia, São Paulo -SP, Brasil \\ p.roberto18@hotmail.com \\ Zulimar Márita Ribeiro Rodrigues \\ Universidade Federal do Maranhão, Departamento de Geociências, Maranhão MA, Brasil \\ zmarita@usp.br \\ Audivan Ribeiro Garcês Júnior \\ Universidade Federal do Ceará, Departamento de Geografia \\ Programa de Pós-Graduação em Geografia, Fortaleza CE, Brasil \\ audivanribeiro@gmail.com \\ Taíssa Caroline Silva Rodrigues \\ Universidade Estadual Paulista, Faculdade de Ciência e Tecnologia \\ Programa de Pós-Graduação em Geografia, São Paulo -SP, Brasil \\ taissageo@hotmail.com
}

\section{RESUMO}

No Brasil, diversos sistemas de indicadores municipais foram desenvolvidos e consolidados através das escalas interurbana e intraurbana. Parte-se do pressuposto que ambas escalas podem e devem ser aplicadas concomitantemente; para uma análise mais acurada das medidas urbanas. O objetivo deste trabalho foi discutir a escala intermunicipal e adaptar à intramunicipal em São Luís-MA, tendo como suporte o Índice de Bem-estar Urbano - IBEU Municipal, já proposto por outros autores. O estudo é descritivo e quantitativo, baseado em dados secundários do censo IBGE 2010, referentes os indicadores dos domicílios e seu entorno. Para análise da escala intraurbana de São Luís, foram avaliadas três dimensões: Condições Ambientais Urbanas (D1), Serviços Coletivos Urbanos (D2) e Infraestrutura Urbana (D3). A dimensão Infraestrutura Urbana (D3) obteve a pior classificação com 0.511 ; seguida de Serviços Coletivos Urbanos (D2) e Condições Ambientais Urbanas (D1), respectivamente, com 0,728 e 0,637 ; valores que representam baixa cobertura dos indicadores que compõe as dimensões. Os piores indicadores que compuseram as dimensões foram: rampa para cadeirantes, bueiro/boca-de-lobo no entorno, arborização e rede de esgotos. A análise das dimensões propostas e seus respectivos, indicadores, mostraram como São Luís é espacialmente desigual na distribuição do bem-estar urbano.

Palavras-Chave: Indicadores. IBEU. Desigualdades socioambientais. Qualidade do ambiente. Área urbana.

\section{INTRA-URBAN INDEX OF WELL-BEING IN SÃO LUÍS-MA, BRAZIL}

\begin{abstract}
In Brazil, several systems of municipal indicators were developed and consolidated through the inter-urban and intra-urban scales. It is assumed that both scales can and should be applied concomitantly for a more accurate analysis of urban measures. The objective of this paper is to discuss the intermunicipal scale and to adapt the intramunicipal in São Luís-MA having as support the Urban Welfare Index - IBEU Municipal, already proposed by other authors. The study is descriptive and quantitative, based on secondary data from the IBGE 2010 census, referring to household indicators and their environment. In order to analyze the intraurban scale of São Luís, three dimensions were performed: Urban Environmental Conditions (D1), Urban Collective Services (D2) and Urban Infrastructure (D3). The dimension Urban Infrastructure (D3) obtained the worst classification with 0.511 , followed by Urban Collective Services (D2) and Urban Environmental Conditions (D1), respectively, with 0.728 and 0.637 , values that represent low coverage of the indicators that make up the
\end{abstract}


dimensions. The worst indicators that made up the dimensions were: ramp for wheelchairs, manhole/grating in the surroundings, afforestation and sewage network. The analysis of the proposed dimensions and their respective indicators have shown how São Luis is spatially unequal in the distribution of urban well-being.

Keywords: Indicators. IBEU. Social and environmental inequalities. Quality of the environment. Urban area.

\section{INTRODUÇÃO}

No Brasil, o desenvolvimento dos indicadores urbanos foi impulsionado pela Constituição de 1988 que propiciou a descentralização, autonomia administrativa e tributária aos municípios, criando assim, uma demanda por informações e/ou indicadores municipais (JANUZZI, 2006; SOLIGO, 2012). Importantes experiências brasileiras podem ser mencionadas como a parceira ocorrida entre o PNUD, IPEA e a Fundação João Pinheiro, podem ser mencionadas, quando lançaram em 1998, dois novos índices para o Brasil: o Índice de Desenvolvimento Humano Municipal - IDHM e o Índice de Condições de Vida - ICV (KOGA, 2003).

Conforme Nahas (2009), no Brasil, entre os anos de 1995 e 2004, diversos sistemas de indicadores sociais municipais foram desenvolvidos, consolidando, portanto, dois momentos: a primeira, a partir de 1990, denominados sistemas de indicadores ou índices intermunicipais e o segundo, consolidados a partir de 1996, denominados intraurbanos. É importante assinalar que os "indicadores interurbanos ou intermunicipais são medidas adotadas com caráter comparativo dos diversos aspectos de uma ou mais cidades. São formas de medir as suas condições econômicas, sociais e territoriais". Na escala intraurbana, o objetivo é avaliar ou medir a dinâmica e/ou desigualdade interna, em sub-regiões da cidade (RODRIGUES, 2010, p.81).

A literatura que aborda os temas índices intermunicipais e intramunicipais menciona as vantagens e desvantagens da utilização das respectivas escalas. Para Koga (2003) com o uso da metodologia intermunicipal é possível realizar comparações e/ou hierarquizações entre as cidades e apreciar a uma série histórica, como é o caso do IDHM. Nahas (2009) também chama atenção para as vantagens das comparações de forma ágil e integrada.

Veiga (2006, p.91) adverte sobre a cautela no uso dos indicadores intermunicipais; afirma que: "[...] é temerário tirar conclusões sobre o desempenho dos municípios a partir de simplórias comparações de IDH-M". Pereira, Cutrim Junior e Rodrigues (2015), interpretam o uso dos índices ou indicadores, como um avanço metodológico e empírico, assim como a transição da escala intermunicipal a intramunicipal em São Luís - MA.

Rodrigues (2010, p.216) argumenta que os índices ou "indicadores intermunicipais e intramunicipais devem ser avaliados de forma concomitante"; pois o primeiro produz a avaliação homogênea e não inferem as desigualdades interna das cidades; e o segundo, o faz. Assim, parte-se do pressuposto que as escalas inter e intramunicipal podem e devem ser aplicadas concomitantemente; para uma análise mais acurada das medidas urbanas. O IBEU Municipal é um índice intermunicipal que avaliou a dimensão urbana do bem-estar para os 5.575 municípios brasileiros, conforme os dados do Censo 2010.

Ribeiro e Ribeiro (2016) afirmam que o IBEU Municipal tem como finalidade a avaliação das condições coletivas de vida no ambiente construído da cidade, levando em consideração a habitação e do seu entorno. Serve como ferramenta para avaliação das condições socioambientais dos municípios e instrumento para o planejamento estatal voltado para a aplicação de políticas públicas de desenvolvimento social e melhoria da qualidade de vida das cidades.

Desse modo, o presente artigo teve como objetivo avaliar a desigualdade intramunicipal em São LuísMA, tendo como suporte a metodologia do IBEU Municipal, proposto por Ribeiro e Ribeiro (2016). Tendo como hipótese que: o crescimento urbano sem planejamento, ocasionou má distribuição de equipamentos e infraestrutura e a escala intraurbana revela estas desigualdades. 


\section{MATERIAIS E MÉTODOS}

\section{Caracterização da área de estudo}

O município de São Luís (Figura 1) se localiza na llha do Maranhão, limitando-se a norte com o oceano Atlântico; ao sul como os municípios de Bacabeira, Rosário e Cajapió; a oeste com Alcântara e Bacurituba e a leste com São José de Ribamar. Pertence a Mesorregião: Norte Maranhense e Microrregião Aglomeração Urbana de São Luís, ocupando mais da metade da ilha (57\%), onde estão também os municípios de São José de Ribamar, Paço do Lumiar e Raposa (IMESC, 2011).

Figura 1: Localização da área de estudo.
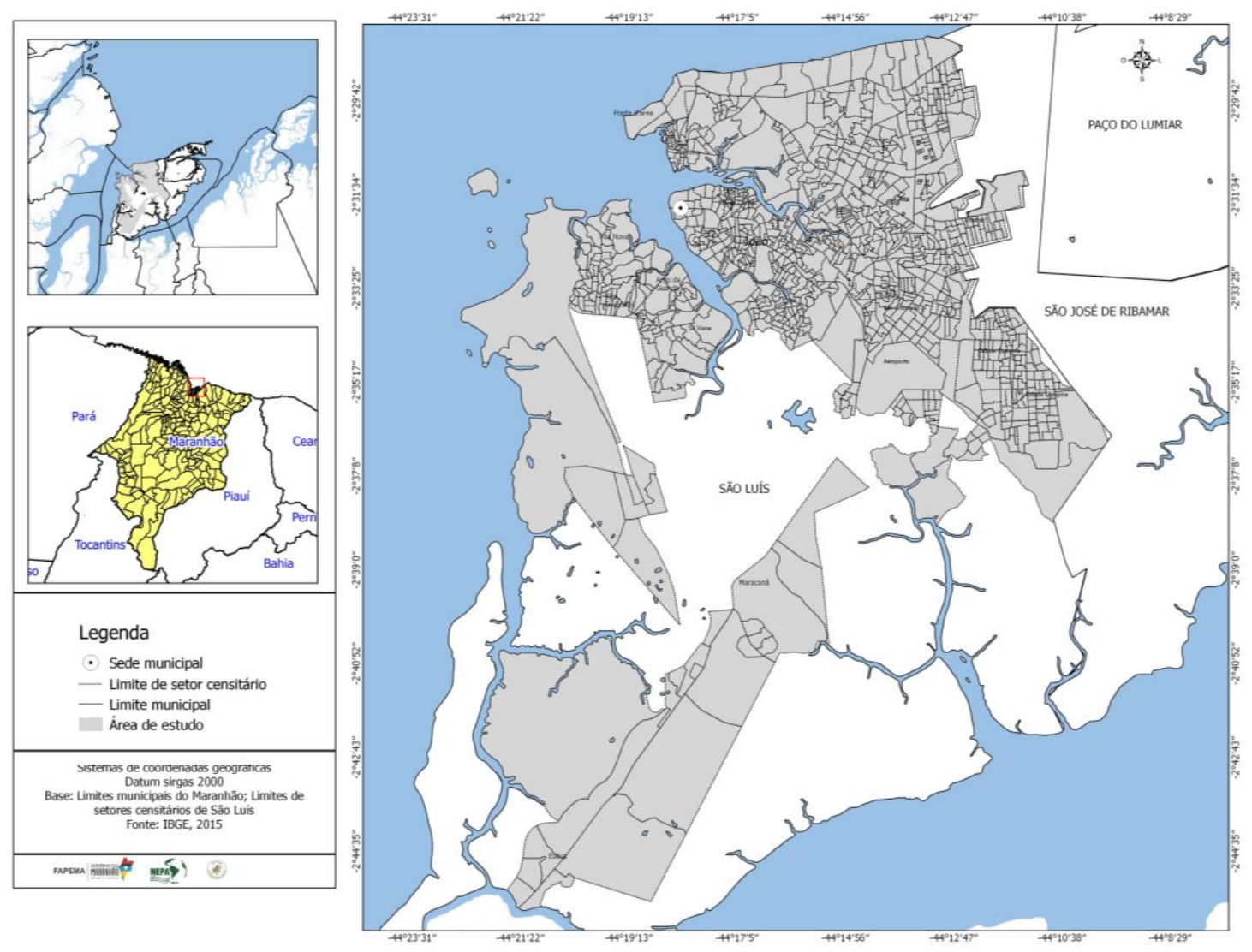

Adaptado de IBGE, 2015.

Fonte:

De acordo com Brasil (2011) o município de São Luís possui área de 834 km², 1.014 .837 habitantes e densidade demográfica de aproximadamente $1.215,69 \mathrm{hab} / \mathrm{km}^{2}$, sendo que $94,45 \%$ da população residem na área urbana. Na Figura 2 se ilustra a densidade demográfica, onde se pode destacar a concentração da população nos bairros periféricos do município, com alta densidade foram representados Vila Embratel, Anjo da Guarda, Vila Nova Sá Viana, Gapara, Fumacê, Vila Mauro Fecury I e II, e Alto da Esperança, Cidade Olímpica. Se destaca também elevada concentração populacional nos grandes conjuntos habitacionais, sendo estes Bequimão, Cohama, Turu, COHAB, COHATRAC e Cidade Operária, com mais de 10.000 habitantes por km² (Figura 2). 
Figura 2: Densidade Demográfica da área urbana de São Luís.

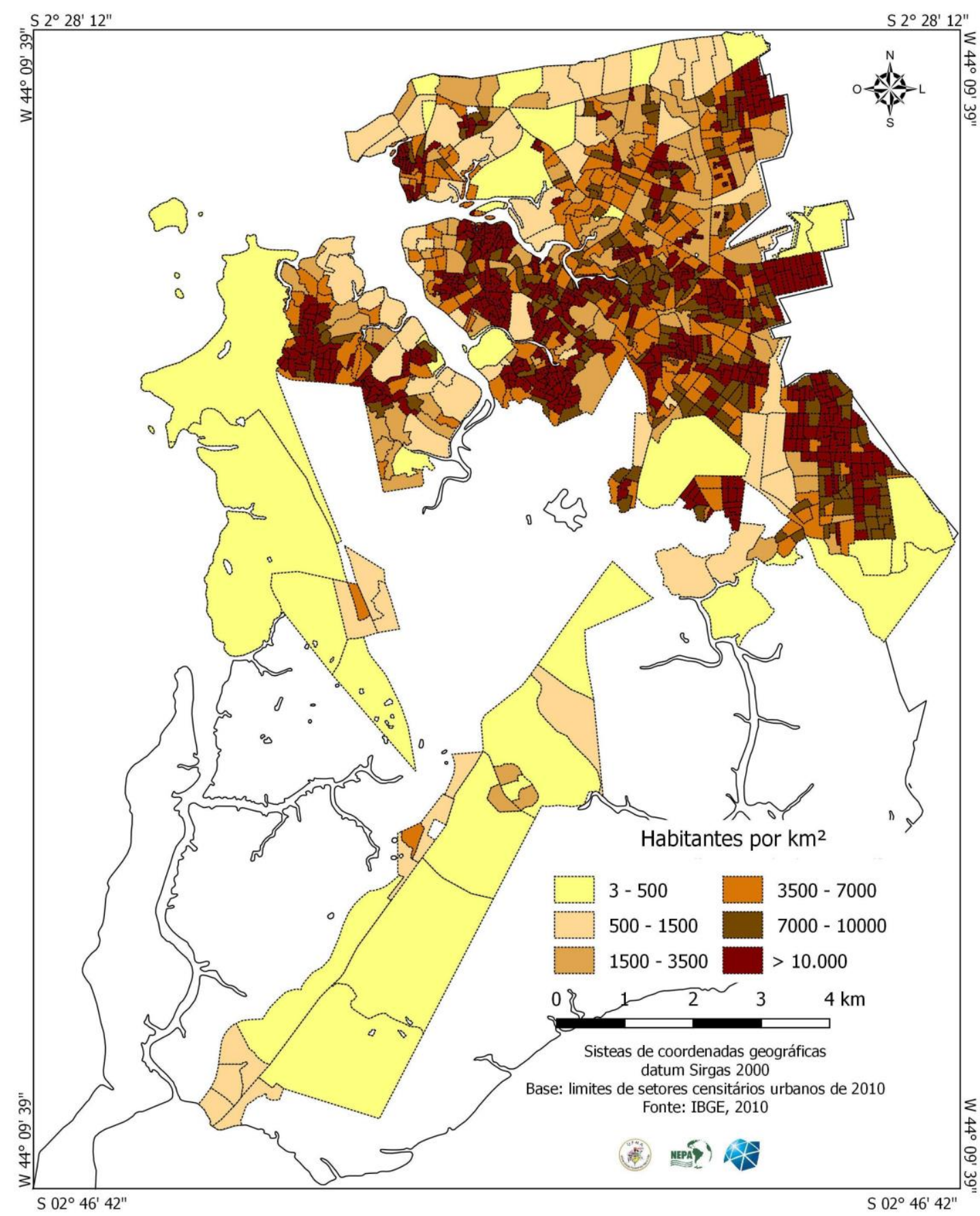

Adaptado do IBGE, 2015.

Fonte:

Ferreira (1999) ao abordar o processo de expansão da capital maranhense, destacou a década 1970, quando houve aumento populacional associado à fixação de indústrias minero-metalúrgicas e a construção da Barragem do Bacanga, que interligou o centro da cidade com a parte sul. Esse aspecto estimulou a ocupação de novos espaços, atualmente denominada área Itaqui-Bacanga, resultante do conglomerado de vários bairros periféricos e sem planejamento prévio. Inerente a dinâmica urbana, São Luís continua em processo de expansão sendo direciona para as áreas mais distantes do centro, correspondendo aos setores rurais que estão sujeitos a especulação imobiliária e/ou expansão industrial

$\begin{array}{llllll}\text { Caminhos de Geografia } & \text { Uberlândia - MG } & \text { v. 19, n. } 67 & \text { Set/2018 } & \text { p. 205-218 Página } 208\end{array}$




\section{Procedimentos}

O trabalho foi realizado a partir do Índice de bem-estar urbano dos municípios brasileiros - IBEU Municipal, proposto por Ribeiro e Ribeiro (2016); com a chancela do Observatório das Metrópoles. As dimensões avaliadas, pelo IBEU Municipal, estão relacionadas "[...] com as condições coletivas de vida promovidas pelo ambiente construído da cidade, nas escalas da habitação e da sua vizinhança próxima, e pelos equipamentos e serviços urbanos" (RIBEIRO e RIBEIRO, 2016, p.01).

O presente estudo é descritivo e quantitativo com a compilação e espacialização dos dados secundário oriundos do censo demográfico de 2010 disponibilizados pelo Instituto Brasileiro de Geografia e Estatística (IBGE). Inicialmente utilizou-se o IBEU Municipal, para comparar a escala intermunicipal de São Luís, dentre as capitais brasileiras.

O IBEU-Municipal é composto por cinco dimensões: mobilidade Urbana, condições Ambientais Urbanas, Condições Habitacionais Urbanas, Atendimento de Serviços Coletivos Urbanos e Infraestrutura Urbana. Cada uma dessas dimensões é composta por número diferente de variáveis, mas todas elas contribuem com o mesmo peso para a definição do IBEU-Municipal (RIBEIRO e RIBEIRO, 2016, p.01).

As cinco dimensões (D) são compostas por diferentes números de indicadores, a saber: mobilidade urbana (D1) (Um indicador - deslocamento casa-trabalho); condições ambientais urbanas (D2) (Três indicadores - arborização do entorno dos domicílios, esgoto a céu aberto no entorno dos domićlios e lixo acumulado no entorno dos domicílios); condições habitacionais urbanas (D3) (Cinco indicadores aglomerado subnormal, densidade domiciliar, densidade morador/banheiro, material das paredes dos domicílios e espécie do domicílio); serviços coletivos urbanos (D4) (Quatro indicadores - atendimento adequado de água, atendimento adequado de esgoto, atendimento adequado de energia e coleta adequada de lixo) e infraestrutura urbana (D5) (Sete indicadores - lluminação pública, pavimentação, calçada, meio-fio/guia, bueiro ou boca de lobo, rampa para cadeirantes e logradouros) (RIBEIRO e RIBEIRO, 2016).

Posteriormente, adaptou-se o IBEU Municipal para a escala intraurbana de São Luís, com a seleção de doze variáveis referentes às características dos domicílios e seu entorno, retirada da planilha de domicílio do censo IBGE 2010. Utilizou-se o software Libre Oficce ${ }^{\circledR}$, sendo a plataforma Calc para os cálculos da definição do melhor e pior valor de cobertura, média, desvio padrão, variância e covariância. Para cada indicador foi utilizada a frequência mínima e máxima de domicílios atendidos pelas variáveis analisadas. Os dados foram padronizados com intervalos entre zero e um, de acordo com a porcentagem de domicílios atendidos, como descrito na equação abaixo:

$$
\text { indicador }=\frac{\text { (valor observado })- \text { (pior valor })}{\text { (melhor valor })- \text { (pior valor) }}
$$

Desse modo o Índice Ambiental Intraurbano de bem-estar em São Luís foi composto por três dimensões: Condições Ambientais Urbanas (D1), Serviços Coletivos Urbanos (D2) e Infraestrutura Urbana (D3), A escolha destas três dimensões levou em consideração a disponibilidades de indicadores para compor cada dimensão, sendo que ao contrário do IBEU original, as variáveis disponíveis não contemplam a dimensão mobilidade urbana, e condições da habitação não sendo realizada para o município estudado. Como fator de adequação do índice geral, todas as dimensões apresentaram peso geral de 1 (um), garantindo assim a igual importância na composição final. A definição de cada dimensão considerou o nível de cobertura das variáveis associadas seguindo a fórmula abaixo, proposta por Ribeiro e Ribeiro (2013) (equação 2).

$$
D=\operatorname{Ind} 1 * P+\operatorname{Ind} 2 * P+(\operatorname{Ind} 3 * P)
$$

Onde: $D=$ dimensão; $P=$ peso atribuído, respectivamente a cada dimensão

A tabela 1 apresenta o peso fatorial que cada indicador possui na formação e nas respectivas dimensões; bem como na composição geral do índice. Os indicadores utilizados foram padronizados de acordo com a média de acesso de domicílios cobertos por indicador e dimensão, sendo os pesos calculados de acordo com o originalmente proposto.

$\begin{array}{llllll}\text { Caminhos de Geografia } & \text { Uberlândia - MG } & \text { v. 19, n. } 67 & \text { Set/2018 } & \text { p. 205-218 } & \text { Página } 209\end{array}$


Tabela 1: Peso dos indicadores e das dimensões na elaboração do índice.

\begin{tabular}{l|l|l}
\hline Dimensões e indicadores & PI & PD \\
\hline Condições De entorno (D1) & $\mathbf{1}$ & $\mathbf{1 / 3}$ \\
\hline Arborização no entorno & $1 / 3$ & $1 / 9$ \\
\hline Ausência de esgoto a céu aberto & $1 / 3$ & $1 / 9$ \\
\hline Ausência de lixo acumulado no entorno & $1 / 3$ & $1 / 9$ \\
\hline Serviços Coletivos (D2) & $\mathbf{1}$ & $\mathbf{1 / 3}$ \\
\hline Abastecimento de água & $1 / 4$ & $1 / 12$ \\
\hline Coleta de lixo & $1 / 4$ & $1 / 12$ \\
\hline Rede de esgoto & $2 / 4$ & $1 / 6$ \\
\hline Infraestrutura do Entorno (D3) & $\mathbf{1}$ & $\mathbf{1 / 3}$ \\
\hline lluminação no entorno & $1 / 6$ & $1 / 18$ \\
\hline Pavimentação no entorno & $1 / 6$ & $1 / 18$ \\
\hline Calçada no entorno & $1 / 6$ & $1 / 18$ \\
\hline Meio-fio no entorno & $1 / 6$ & $1 / 18$ \\
\hline Presença de bueiro/boca-de-lobo no entorno & $1 / 6$ & $1 / 18$ \\
\hline Presença de rampa para cadeirante no entorno & $1 / 6$ & $1 / 18$ \\
\hline
\end{tabular}

Fonte: dados de pesquisa.

Para o fator de confiabilidade do agrupamento, utilizou-se o coeficiente Alfa de Cronbach (equação 3) que trabalha a relação entre covariâncias e variâncias internas das dimensões e procura estabelecer o relacionamento entre elas variando de zero a um (tabela 2). Quanto mais próximo de um, melhor é o grau de relacionamentos entre as dimensões; quanto mais próximo de zero, menor é o grau de relacionamento sendo calculado de acordo com a equação abaixo (PEREIRA, 2001).

$$
\alpha=\frac{k *\left(\frac{\operatorname{Cov}}{V a r}\right)}{1+(K-1) *\left(\frac{c O V}{V a r}\right)}
$$

Onde: $\alpha=$ Alfa de Cronbach; $K$ = número de variáveis consideradas; cov = Média das covariâncias; var = Média das variâncias.

Tabela 2: Estatística descritiva e Correlação geral das dimensões.

\begin{tabular}{c|c|c|c|c|c|c}
\hline Dimensões & Média & Variância & $\begin{array}{c}\text { Desvio } \\
\text { Padrão }\end{array}$ & Correlação & Alfa & № de variáveis \\
\hline D1 & 1,82 & 0,48 & 0,69 & 0,482 & 0,501 & 3 \\
\hline D2 & 3,05 & 1,68 & 1,29 & 0,781 & 0,761 & 3 \\
\hline D3 & 2,2 & 0,47 & 0,69 & 0,610 & 0,646 & 6 \\
\hline
\end{tabular}

Fonte: dados de pesquisa.

A base cartográfica foi selecionada a partir dos limites de setores censitários urbanos, no total de 1.051 (IBGE, 2010), aqui classificadas em quatro áreas tendo como fator limitante o percentil dos valores dos índices por setor, e tendo como base ainda a classificação proposta por Rodrigues (2010), conforme tabela 3.

Tabela 3: Classificação das áreas para o Índice Ambiental Intraurbano.

\begin{tabular}{l|l|c}
\hline \multicolumn{1}{c|}{ Classes das áreas } & Intervalos & № de setores censitários agrupados \\
\hline Bom & $0,751-0,942$ & 275 \\
\hline Regular & $0,501-0,750$ & 451 \\
\hline Ruim & $0,251-0,500$ & 266 \\
\hline Péssimo & $0,009-0,250$ & 59 \\
\hline
\end{tabular}

Fonte: Dados de pesquisa.

$\begin{array}{llllll}\text { Caminhos de Geografia } & \text { Uberlândia - MG } & \text { v. 19, n. } 67 & \text { Set/2018 } & \text { p. 205-218 } & \text { Página } 210\end{array}$




\section{RESULTADOS E DISCUSSÃO}

\section{CLASSIFICAÇÃO DE SÃO LUÍS NA ESCALA INTERMUNICIPAL DO IBEU}

São Luís, capital do estado do Maranhão, caracteriza-se como espaço dinâmico com diferenças internas e desigualdades socioambientais como observadas por Rodrigues (2010) e Rodrigues e Pereira (2014), estando associado diretamente ao crescimento urbano acelerado, observados nos últimos 40 anos, resultado da deficiência e/ou ineficiência da distribuição de serviços equipamentos urbanos básicos e precariedade do sistema de saneamento para todo município.

Segundo o Índice de bem-estar urbano dos municípios brasileiros - IBEU Municipal, em 2016 o IBEUMunicipal de São Luís foi de 0,700 , classificada como condições "ruins" de bem-estar urbano. Entre as capitais obteve a $22^{\circ}$ posição; entre todos os municípios brasileiros, a $4.493^{\circ}$ posição. Cabe esclarecer que para o IBEU Municipal, os municípios brasileiros podem ser classificados, segundo os intervalos: "de 0,00 a 0,500 corresponde às condições muito ruins; de 0,501 a 0,700 corresponde às condições ruins; de 0,701 a 0,800 corresponde às condições médias; de 0,801 a 0,900 corresponde às condições boas; de 0,901 a 1 corresponde às condições muito boas" (RIBEIRO e RIBEIRO, 2016, p.5).

Desde modo, a classificação calculada para São Luís, é referente as cinco dimensões e comparada entre as capitais e demais municípios de acordo com os dados de Ribeiro e Ribeiro (2016). No âmbito das dimensões, a "mobilidade urbana (D1)", foi a melhor avaliada, pois a capital maranhense obteve 0,832 e foi considerada em "boas condições". Entre as capitais, está na $21^{1 \underline{a}}$ posição, sendo Boa Vista (RR) em primeiro, como "muito boa"; e São Paulo (SP), na $27^{\circ}$ posição, considerada em condições "ruins". A mobilidade urbana foi interpretada através do indicador deslocamento casa-trabalho, "utiliza-se a proporção de pessoas ocupadas que trabalham fora do domicílio e retornam para casa diariamente que gastam até 1 hora no trajeto casa-trabalho" (RIBEIRO e RIBEIRO, p. 3, 2016).

A dimensão "condições habitacionais urbanas (D3)" e "serviços coletivos urbanos (D4)", foram classificadas como em níveis "médios" de bem-estar. Na primeira, São Luís pontuou 0,754 e foi ranqueada em $24^{\circ}$ lugar. Florianópolis (SC) em 1ํe e Belém (PA) em 27으, apontadas, respectivamente, como níveis "muito bom" e "ruim". Na segunda dimensão, a capital maranhense, pontou 0,728 e foi ranqueada em 18; sendo Vitória (ES) em primeiro e Porto Velho $(\mathrm{RO})$ em último, avaliadas, respectivamente, como "muito bom" e "ruim" (RIBEIRO E RIBEIRO, 2016).

Para a dimensão "condições ambientais urbanas", identificou-se como indicador pior avaliado a "arborização no entorno", um claro sinal ou alerta sobre a ausência de áreas verdes em São Luís. Ainda que, distribuída de forma desigual, a arborização está mais presente nas áreas classificadas como péssimas. O melhor indicador avaliado foi ausência de lixo acumulado no entorno dos domicílios; sobretudo nas áreas classificados como bom. Entretanto, esse indicador aparece nas áreas consideradas ruim e péssimas.

As dimensões "condições ambientais urbanas (D2)" e "infraestrutura urbana(D5)" foram as duas piores avaliadas, apontadas como "ruins". São Luís pontuou, respectivamente, 0.637 e 0.551 e classificada em 23ํㅡ e 21‥ Na primeira dimensão em 1ำ lugar ficou Campo Grande (MS), com 0.965 e em último Belém (PA), com 0,549. Na segunda, Macapá (AP) com 0,367 e Vitória (ES) com 0,817. Assim, entre a cinco dimensões proposta pelo IBEU-Municipal, São Luís foi pior avaliada para "infraestrutura urbana (D5)", com 0.551.

\section{Índice Infraestrutura urbana proposto para São Luís}

O índice de bem-estar intraurbano foi composto em três dimensões: Condições Ambientais Urbanas (D1), Serviços Coletivos Urbanos (D2) e Infraestrutura Urbana (D3). A dimensão Condições Ambientais Urbanas (D1) apresentou $60 \%$ de domicílios com cobertura para os indicadores analisados. Esta dimensão apresentou valores intermediários quanto ao percentual de cada indicador que a compôs. Sendo seu pior indicador arborização no entorno, com apenas $31 \%$ de domicílios com acesso; refletindo a ausência de áreas verdes na cidade. Quanto ao melhor indicador, ausência de lixo no entorno, observado em $86 \%$ dos domicílios, configurando como fator que mais influenciou na composição da dimensão avaliada (Figura 3). 
Figura 3: Mapa de distribuição das variáveis da Dimensão 1 - Condições do entorno.

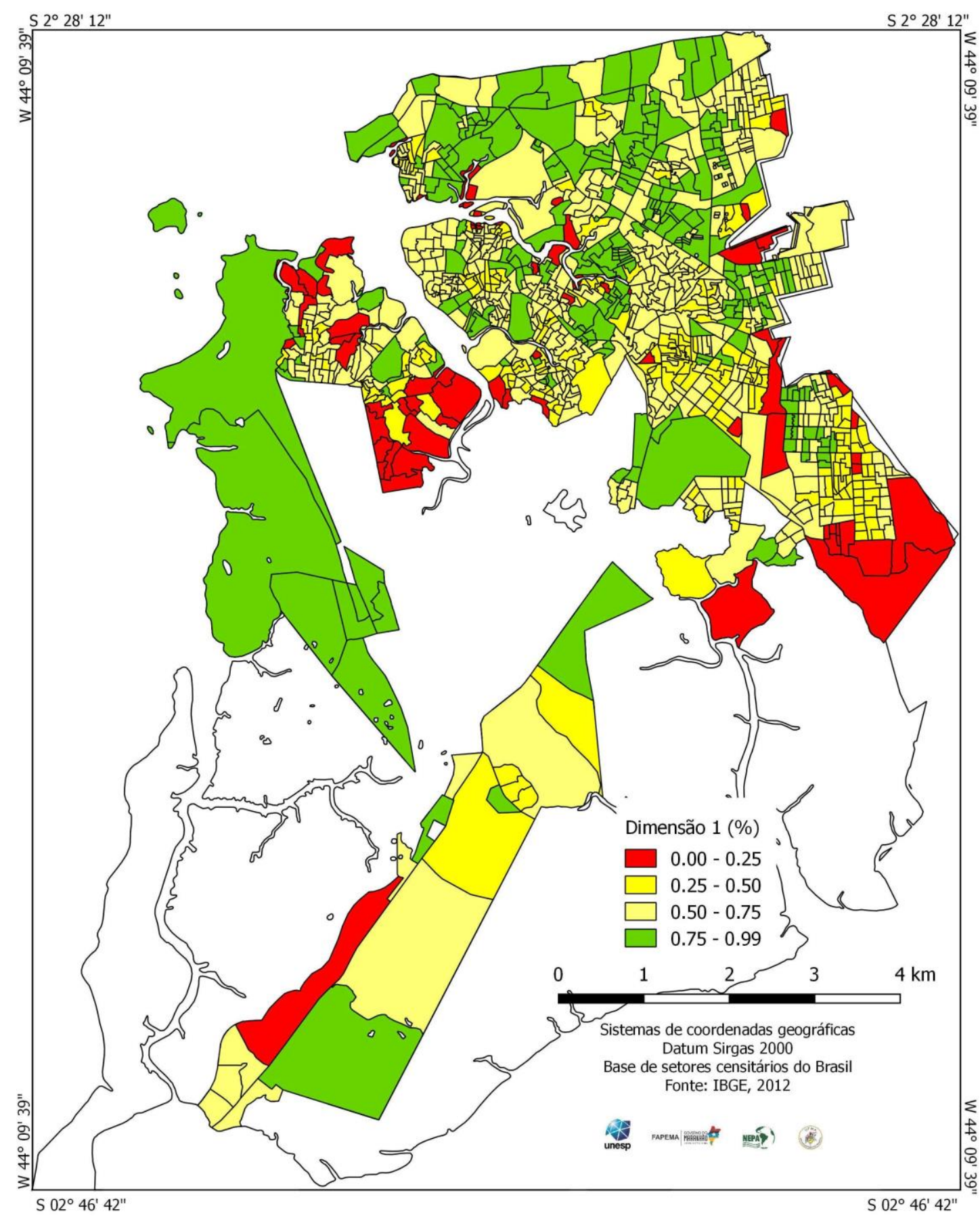

Fonte: Elaboração dos autores.

A dimensão Serviços Coletivos Urbanos (D2) foi a que mais contribuiu para a formação do índice, pois $63 \%$ dos domicílios apresentaram cobertura por esta dimensão. Em relação à composição geral dos indicadores, a coleta de lixo foi o que mais contribuiu para esta dimensão, com uma média geral de $88 \%$ de domicílios com acesso. Em segundo lugar o indicador abastecimento de água da rede geral com 78\%; e o pior indicador, a rede coletora de esgoto com média de apenas $49 \%$ dos domicílios com acesso, mostrando ausência de um sistema de saneamento eficiente e/ou descompasso entre rede de abastecimento de água e rede coleta de esgotos (Figura 4). 
Figura 4: Mapa de distribuição das variáveis da Dimensão 2 - Serviços Coletivos.

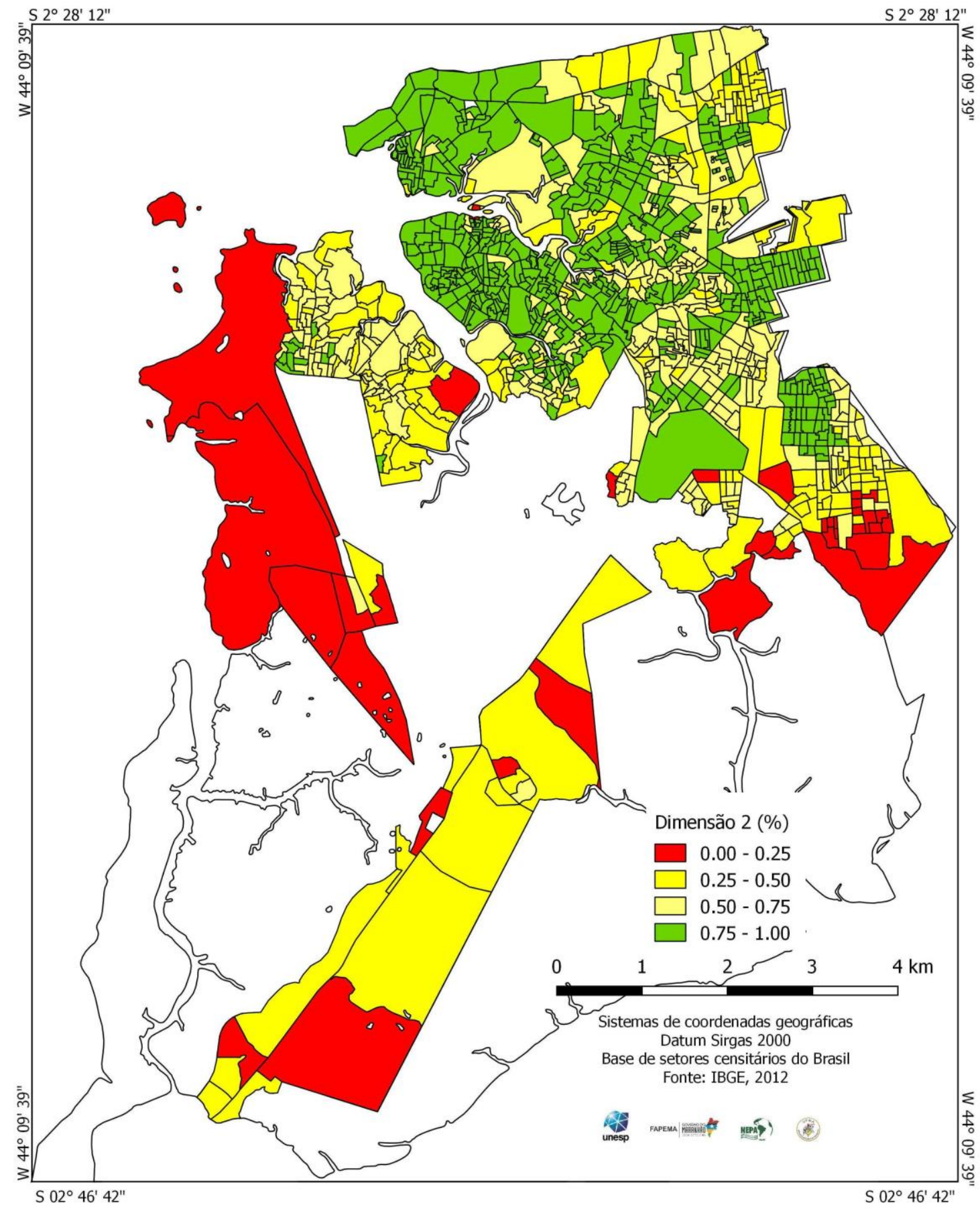

Elaboração dos autores.

Fonte:

A terceira dimensão, buscou analisar as variáveis ao entorno apresentou o segundo melhor padrão de distribuição das variáveis. Na composição geral do índice, a dimensão que menos contribuiu foi a Infraestrutura Urbana (D3) com uma média de $49 \%$ de domicílios com a cobertura dos indicadores analisados, porém apresentou o segundo melhor valor de aceitação de padronização com alfa de cronbach de 0,6. Entre os indicadores que compõem esta dimensão destaca-se que o pior valor foi para presença de rampa para cadeirante no entorno, com apenas $2 \%$ das áreas adjacentes aos domicílios possuindo condições ideais. O melhor indicador para esta dimensão foi a iluminação no entorno com até $89 \%$ dos domicílios sendo assistido por esse equipamento urbano (Figura 5). 
Figura 5: Mapa de distribuição das variáveis da Dimensão 2 - Serviços Coletivos.

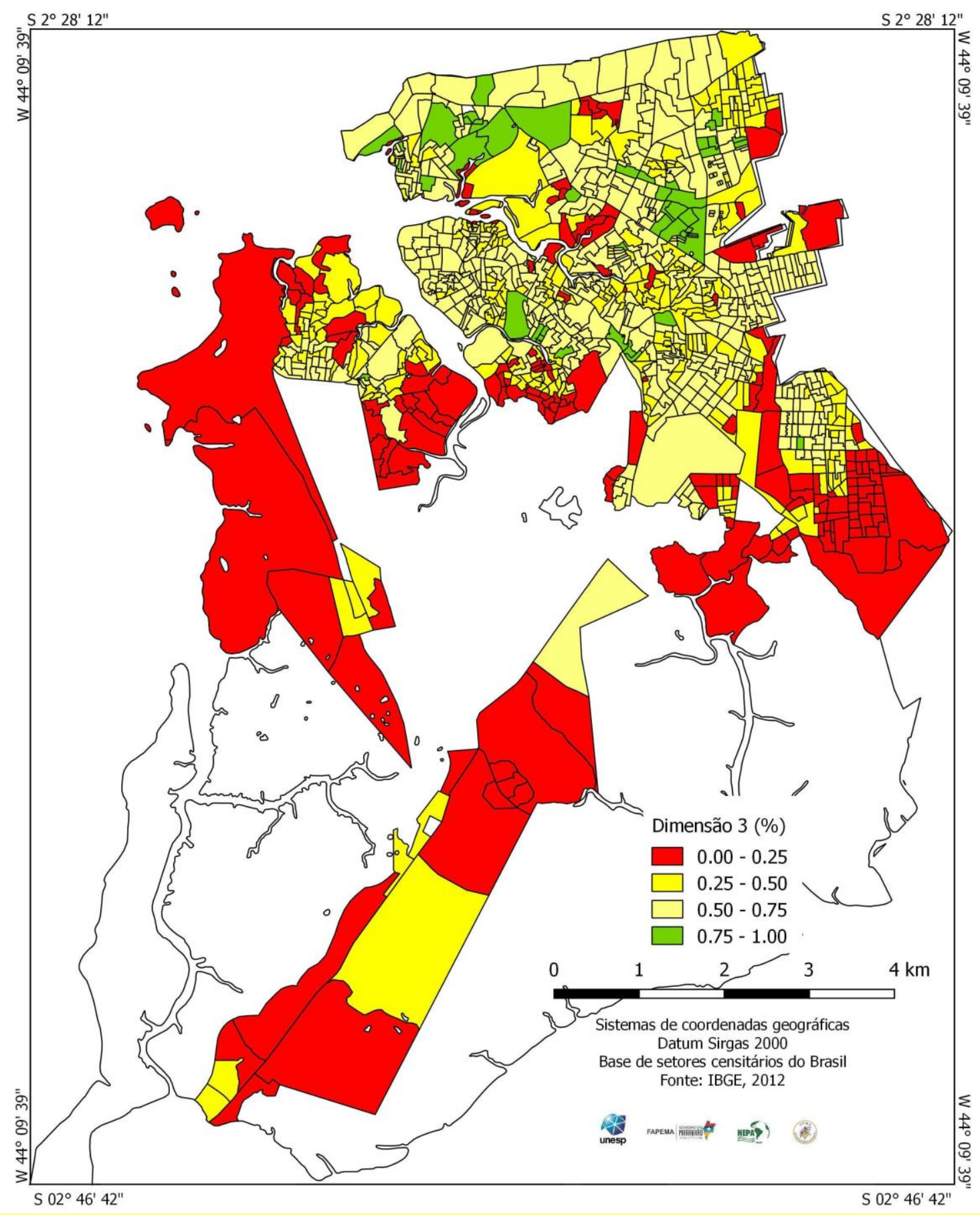

Fonte: Elaboração dos autores.

A dimensão "serviços coletivos urbanos", mesmo sendo a melhor avaliada, apresenta indicadores preocupantes; sobretudo, a "rede de esgoto". Esse indicador contribuiu com apenas $49 \%$ para compor a dimensão e se contrapõe claramente ao abastecimento de água que contribuiu com $78 \%$. Ou seja, o serviço de abastecimento de água é maior que a rede coletora de esgotos. $\mathrm{O}$ indicador melhor avaliado foi coleta de lixo. Novamente a dimensão relevou a desigualdade intraurbana, pois os serviços de abastecimento de água, coleta de lixo e rede de esgotos têm maiores percentuais nas áreas classificadas com bom. Desse modo, pode-se destacar que as áreas identificadas como "BOM" correspondem a áreas significativamente menores quando \begin{tabular}{llllll}
\hline Caminhos de Geografia & Uberlândia - MG & v. 19, n. 67 & Set/2018 & p. 205-218 & Página 214
\end{tabular} 
comparados as áreas "REGULAR", "RUIM" e "PÉSSIMO", esse aspecto caracteriza a desigualdade intraurbana. As áreas classificadas como "BOM" têm os melhores níveis de coberturas das três dimensões avaliadas, entretanto distribuídas em menores proporções na cidade

Para comparação do Índice Ambiental Intraurbano de São Luís foram consideradas quatro classes de áreas: "BOM", "REGULAR", "RUIM" e "PÉSSIMO". As áreas representadas pelos melhores resultados, classificadas como "BOM", formaram o segundo maior aglomerado de setores censitários, com 275. Caracterizada por bairros que apresentam algum planejamento em sua formação, os conjuntos habitacionais, tais como: Vinhais, COHAB, COHAMA, COHATRAC, Vinhais e Cidade Operária. Participaram ainda os bairros mais tradicionais e/ou considerados de classe média ou alta, como São Francisco, Renascença, Ponta d'Areia, São Marcos e Angelim. No geral, esta área representa os setores censitários com os melhores valores para a cobertura das três dimensões avaliada, entretanto com a menor extensão territorial da cidade (Figura 6).

A área "REGULAR" foi constituída por 451 setores, refletindo o primeiro maior agrupamento. Caracteriza-se por agrupar os bairros mais antigos, abrangendo principalmente o Centro, João Paulo, Monte Castelo, grande parte Sacavém e Vila Palmeira. Entre as três dimensões, Serviços Coletivos Urbanos (D2) e Infraestrutura Urbana (D3) têm a melhor cobertura; e as Condições Ambientais Urbanas (D1), a pior.

As áreas classificadas como "RUIM" agrupou 266 setores, sendo o terceiro grupo. A área é composta pelos bairros do Anil, Turu, Santo Antônio, Santa Rosa, e pequena extensão da área Itaqui-Bacanga. Uma peculiaridade da área é a proximidade e/ou interposição, com as áreas "BOAS" e "REGULARES", que dispõem de melhores condições avaliadas. Assim, essa área constitui-se uma heterogeneidade das dimensões entre setores censitários que compõe os bairros. Entre as dimensões, as Condições Ambientais Urbanas (D1) contribuiu entre $60 \%$ e $75 \%$ de domicílios com acesso. Os Serviços Coletivos Urbanos (D2) e Infraestrutura Urbana (D3) apresentaram valores intermediários, tendo entre $50 \%$ e $55 \%$ de domicílios com cobertura das dimensões.

As áreas caracterizadas como "PÉSSIMA" foi composta por 59 setores censitários. Em contrapartida representa as áreas compostas por setores censitários de grande extensão territorial e abrange os bairros mais distantes do Centro, cujas formações originais são áreas de ocupações desordenadas, com destaque para Cidade Olímpica, Jardim América, Coroadinho, Sacavém, Divineia e grande extensão da área Itaqui-Bacanga; estas áreas também se caracteriza por apresentar as maiores densidades populacionais. Quanto a associação das dimensões para estas áreas, destaca-se que nenhuma das três dimensões apresentou valores superiores a $25 \%$ de domicílios atendidos pelos indicadores que as constitui, um dado observado para estas áreas é a dissimilaridade entre o sistema de abastecimento e rede coletora de água

As análises mostram a incompatibilidade de distribuição das variáveis analisadas por dimensão. O abastecimento de água pode ser considerado um dos melhores serviços, atingindo um percentual $80 \%$ dos setores agrupando 206.156 domicílios distribuídos nos 1052 setores censitários analisados. Cabe destacar que a variação entre as áreas boas e péssimas para a rede de esgoto foi de aproximadamente 8 vezes melhor, e a distribuição de água foi de até 1,5 vezes melhor.

Ainda assim, é oportuno lembrar que há desigualdade na oferta dos serviços, considerando que o sistema de abastecimento da água na cidade é intermitente, há mais de cinco anos consecutivos, sobretudo, nas áreas com moradores de baixo poder aquisitivo. A distribuição da rede de esgoto pode ser considerada o serviço com o pior percentual, atingindo, aproximadamente, 128.960 domicílios ou apenas $49,14 \%$ dos setores censitários analisados, na área existe apenas duas estações de tratamento de esgoto domésticos, que não funcionam com capacidade total. Gerenciada pela Companhia de Saneamento Ambiental do Maranhão - CAEMA. Esse fator pode explicar o alto grau de lançamento de resíduos domésticos líquidos in natura em rios e na orla marítima do município. 
Figura 6 - Índice Ambiental Urbano de São Luís.

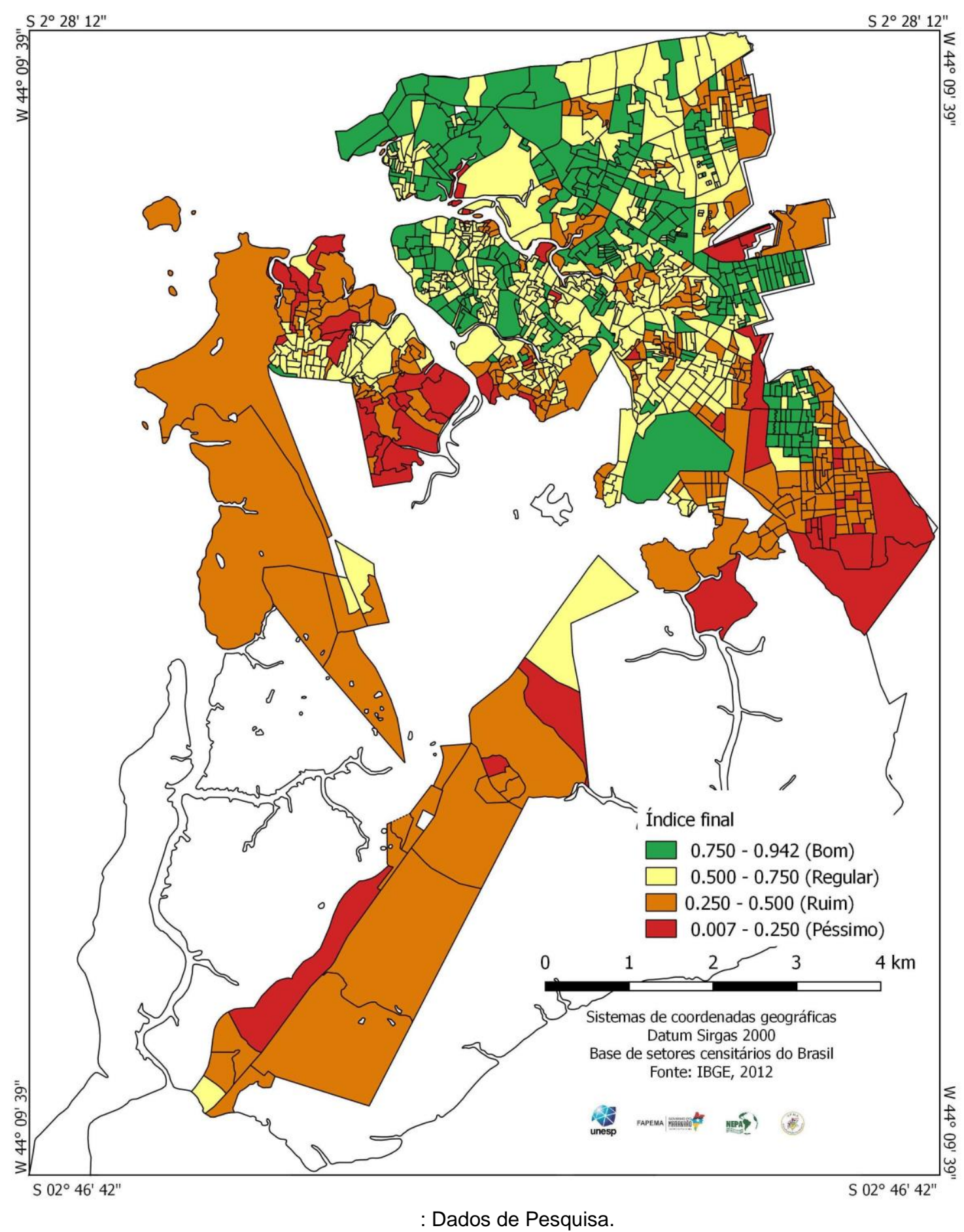

Fonte

\section{CONSIDERAÇÕES FINAIS}

Mapear a distribuição de serviços e infraestrutura urbana revelou como São Luís é espacialmente desigual. A proposta de adaptação do índice de Bem-Estar Urbano Municipal utilizado neste trabalho apresentou-se com uma metodologia complementar, haja vista que foi possível verificar de forma mais detalhada a distribuição desigual das dimensões dentro do município. Parte-se do pressuposto que os índices intermunicipais devem ser complementados com a escala intramunicipal. 
Entre as três dimensões analisadas: "serviços coletivos urbanos", "condições ambientais urbanas" e "infraestrutura urbana"; esta última, foi a pior avaliada, sobretudo, pela ausência de "rampa para cadeirantes no entorno" e de "bueiro/boca-de-lobo no entorno". A ausência de ambos indicadores contribuiu negativamente para a avaliação da dimensão; assim, pode-se inferir que a acessibilidade da cidade é deficitária para um segmento da população e tem problemas de drenagem urbana, principalmente nas áreas classificadas como ruim e péssima.

As áreas consideradas "bom" com melhor infraestrutura e dos serviços urbanos, são pequenas áreas, se comparado com toda a área da cidade. As demais áreas juntas são geograficamente maiores, com déficit de serviços urbanos essenciais.

\section{AGRADECIMENTOS:}

A Fundação de Amparo à Pesquisa e ao Desenvolvimento Científico e Tecnológico do Maranhão FAPEMA, pela concessão da bolsa de estudos. Ao Instituto Brasileiro de Geografia e Estatística subsede São Luís, pela disponibilidade dos dados. Ao Núcleo de Estudos e Pesquisas Ambientais NEPA/UFMA, pelo auxilio nas atividades e pela estrutura disponibilizada para as análises dos dados

\section{REFERENCIAS}

BRASIL, Instituto Brasileiro de Geografia e Estatística. Bases de Informações do Censo Demográfico 2010: Resultados do Universo Por Setor Censitário. Rio de Janeiro: IBGE, 2011.

FERREIRA, Antonio José de Araújo. O estado e as políticas do urbano em São Luís. Dissertação (Mestrado em Geografia) - Faculdade de Filosofia, Letras e Ciências Humanas, Universidade de São Paulo, São Paulo, 1999.

JANNUZZI, Paulo de Martino. Indicadores Sociais no Brasil. Conceitos, fontes de dados e aplicações. Campinas, SP: Editora Alínea, 2006.

IBGE, Instituto Brasileiro de Geografia e Estatística. Censo Demográfico. Rio de Janeiro, 2010. Instituto Brasileiro de Geografia e Estatística. Base de divisão municipal do Brasil. Disponível em www.ibge.bov.br/malhasdigitais. Acesso em 23/02/2017.

IMESC, Instituto Maranhense de Estudos Socioeconômicos e Cartográficos. Situação ambiental da Ilha do Maranhão. São Luís: IMESC, 2011.

KOGA, Dirce. Medidas de cidades: entre territórios de vida e territórios vividos. São Paulo: Cortez, 2003.

NAHAS, Maria Inês Pedrosa.Indicadores Intra-Urbanos como instrumento de gestão da qualidade de vida urbana em grandes cidades: Uma discussão teórico - metodológica. in: VITTE, Claudete de Castro; KEINERT, Tânia Margarete Mezzomo. Qualidade de vida, Planejamento e Gestão Urbana: discussões teórico - metodológicas. Rio de Janeiro: Bertrand Brasil. 2009

PEREIRA, Júlio Cesar Rodrigues. Análise de Dados Qualitativos: Estratégia Metodológicas para as Ciências da Saúde, Humanas e Sociais. $3^{\text {a }}$ Ed. São Paulo: Editora da Universidade de São Paulo, 2001.

PEREIRA, Paulo Roberto Mendes; CUTRIM JUNIOR Valdir; RODRIGUES, Zulimar Márita Ribeiro. Monitoramento da qualidade ambiental intraurbana de São Luís no período intracensitário 2000/2010: indicadores de saneamento e habitação. In: FARIAS FILHO, Marcelino Silva; CELERI, Marcio José (org). Geografia da llha do Maranhão - São Luís, MA. EDUFMA, 2015. 290p. 224-237.

RIBEIRO, Luiz Cesar de Queiroz; RIBEIRO, Marcelo Gomes Ribeiro. (Org.). IBEU: índice de bemestar urbano. 1 ed. Rio de Janeiro: Letra Capital, 2013. Disponível em: http://www.observatoriodasmetropoles.net/index. Acesso em 20/03/2014.

RIBEIRO, Luiz Cesar de Queiroz; RIBEIRO, Marcelo Gomes Ribeiro. (Org.). IBEU MUNICIPAL: índice de bem-estar urbano dos municípios brasileiros. 1 ed. Rio de Janeiro: Letra Capital, 2016. Disponível em: http://www.observatoriodasmetropoles.net/index. Acesso em 25/10/2016 
RODRIGUES. Zulimar Márita Ribeiro. Sistema de indicadores e desigualdade socioambiental intraurbana de São Luís - MA. Tese (Doutorado em Geografia) - Faculdade de Filosofia, Letras e Ciências Humanas, Universidade de São Paulo, São Paulo, 2010.

SOLIGO, Valdecir. Indicadores: conceito e complexidade do mensurar em estudos de fenômenos sociais. In: Est. Aval. Educ., São Paulo, v. 23, n. 52, p. 12-25, mai./ago. 2012. Disponível em: http://www.fcc.org.br/pesquisa/publicacoes/eae/arquivos/1724/1724.pdf

VEIGA, José Eli da. Desenvolvimento sustentável: o desafio do século XXI. 2. ed. Rio de Janeiro: Garamond, 2006.

Recebido em: 25/07/2017

Aceito para publicação em: 08/07/2018 